\title{
The role of the serum RANKL/OPG ratio in the healing of intertrochanteric fractures in elderly patients
}

\author{
XUE-FEI WANG ${ }^{1}$, YA-KUI ZHANG ${ }^{1}$, ZHEN-SHAN YU ${ }^{1}$ and JUN-LIN ZHOU ${ }^{2}$ \\ ${ }^{1}$ Department of Orthopedics, Beijing Chaoyang Hospital, Capital Medical University, Beijing 100020; \\ ${ }^{2}$ Department of Orthopedics, The Luhe Teaching Hospital of the Capital Medical University, Beijing 101149, P.R. China
}

Received November 12, 2012; Accepted February 18, 2013

DOI: $10.3892 / \mathrm{mmr} .2013 .1335$

\begin{abstract}
Intertrochanteric fractures occur most commonly in elderly patients. Osteoblasts and osteoclasts have been reported to be regulated by the receptor activator of $\mathrm{NF}-\kappa \mathrm{B}$ ligand (RANKL) and osteoprotegerin (OPG), during bone modeling and remodeling, respectively. Based on these observations, we hypothesized that the serum levels of RANKL, OPG and the RANKL/OPG ratio are important in the healing of intertrochanteric fractures in elderly patients. Enzyme-linked immunosorbent assays were used to measure the serum concentrations of RANKL and OPG in 36 elderly patients with intertrochanteric fractures and 30 age-matched healthy control subjects, at baseline and 4, 8 and 12 weeks following injury. The RANKL/OPG ratio in the two groups was also evaluated. Similar trends in RANKL and OPG levels were detected during the fracture healing process. The serum levels of RANKL and OPG were higher in the fracture group than in the controls, and were significantly higher at baseline and 4 weeks following injury $(\mathrm{P}<0.05)$. Notably, although the RANKL/OPG ratio gradually increased during healing, it was lower in the fracture group than in the control group. The RANKL/OPG ratio was significantly lower immediately after and 4 weeks after injury in the fracture group $(\mathrm{P}<0.05)$. Our data suggest a close correlation between higher serum levels of RANKL and OPG and the fracture healing process, indicating that RANKL and OPG are involved in fracture healing. The serum RANKL/OPG ratio also appears to be significant in the healing of intertrochanteric fractures in elderly patients.
\end{abstract}

\section{Introduction}

Intertrochanteric fractures encountered by orthopedic surgeons most commonly occur in the elderly population (1). Complications of intertrochanteric fractures, such as nonunion

Correspondence to: Dr Jun-Lin Zhou, Department of Orthopedics, Beijing Chaoyang Hospital, Capital Medical University, Beijing 100020, P.R. China

E-mail: junlinzhou@yahoo.cn

Key words: intertrochanteric fracture, serum, receptor activator of $\mathrm{NF}-\kappa \mathrm{B}$ ligand, osteoprotegerin and delayed healing, are not common during fracture healing due to the good blood supply and excellent cancellous bone in the intertrochanteric area (2). However, complications resulting in pain, functional disability, psychological barriers and an economic burden may occur in patients with intertrochanteric fractures. To date, there have been few studies concerning primary intertrochanteric nonunions and their prevention $(1,3,4)$. Although a number of methods for predicting the progress of fracture healing exist, diagnostic methods at a molecular level are lacking. Bone biochemical markers are likely to be of particular interest, since they reflect the metabolic states of osteoblasts and osteoclasts (5). Changes in these markers may reflect the molecular basis of fracture healing.

Receptor activator $\mathrm{NF}-\kappa \mathrm{B}$ (RANK), receptor activator $\mathrm{NF}-\kappa \mathrm{B}$ ligand (RANKL) and the osteoprotegerin (OPG) molecular system play important roles in the biology of osteoblasts and osteoclasts (6). RANK, a member of the tumor necrosis factor receptor superfamily, is a main activator of the transcription factor $\mathrm{NF}-\kappa \mathrm{B}$, and acts as a receptor for RANKL in a signal transduction pathway. RANKL is secreted by osteoblasts; following binding to RANK on the surface of preosteoclasts, RANKL stimulates the activation and development of osteoclasts. The soluble protein OPG is also a member of the tumor necrosis factor receptor superfamily, and is secreted by many cell types, such as the liver, heart, spleen, kidney cells and osteoblasts. OPG inhibits bone resorption by inhibiting the interaction between RANKL and RANK (7-10).

The RANKL/OPG system is a major signaling pathway that regulates the differentiation and function of osteoblasts and osteoclasts (11). RANKL, which is a cytokine, is synthesized and secreted by osteoblasts and promotes bone resorption. Following binding to RANKL, OPG inhibits bone resorption caused by osteoclasts and increases bone formation $(12,13)$. Therefore, a number of studies have suggested that the balance between RANKL and OPG regulates bone resorption and formation (14-18). However, the changes in RANKL and OPG that occur during fracture healing in elderly patients with intertrochanteric fractures, as well as the importance of the RANKL/OPG ratio, have not yet been elucidated.

In the present study, the changes of serum RANKL and OPG levels in elderly patients with intertrochanteric fractures were investigated over time, and were compared with the respective levels in healthy control subjects. These measure- 
ments were analyzed to investigate the roles of RANKL, OPG and the RANKL/OPG ratio in elderly patients with normal intertrochanteric fracture healing, and to determine the significance of the RANKL/OPG ratio in elderly patients with intertrochanteric fractures.

\section{Patients and methods}

Study subjects. The study group included 36 patients with intertrochanteric fractures, with a mean age of 69.0 years (range, 65-79 years). All patients were treated between January 1, 1998 and January 1, 2005 in the Department of Orthopedics, The Luhe Teaching Hospital of the Capital Medical University. All patients had no systemic diseases and were admitted to the hospital immediately after injury. Surgery was performed on all patients with intertrochanteric fractures by the same group of surgeons. After surgery, all the patients underwent serial radiography to evaluate bone formation. All the fractures were healed 16-26 weeks following surgery. The control group consisted of 30 healthy subjects with a mean age of 71.0 years (range, 65-81 years). The study was approved by the Research Ethics Committees of our hospital, and informed consent was obtained from all study subjects.

Measurement of serum RANKL and OPG levels. Peripheral blood samples $(20 \mathrm{ml})$ were obtained from the 36 patients with intertrochanteric fractures within the first few hours of admission to hospital (at baseline), as well as at 4,8 , and 12 weeks following injury. Additionally, $20 \mathrm{ml}$ peripheral blood were obtained from normal control subjects at baseline and after 4 , 8 and 12 weeks. Serum was extracted from the blood samples in the morning and was immediately centrifuged. All the specimens were stored at $-80^{\circ} \mathrm{C}$ until analyzed.

A sandwich enzyme-linked immunosorbent assay (ELISA; Immundiagnostik, Bensheim, Germany) was used to assess RANKL levels in the serum samples. Firstly, the biotin anti-RANKL detection antibody (sc-377079; Santa Cruz Biotechnology, Inc., Santa Cruz, CA, USA) and the serum sample were pipetted into the wells of the ELISA plate. When human RANKL is present in the serum sample, it binds the recombinant OPG (sc-358754, Santa Cruz Biotechnology) precoated onto the wells and constitutes a sandwich with the detection antibody. In order to remove nonspecifically bound material, a streptavidin-horseradish peroxidase conjugate was added to the wells following washing. After removing the unbound conjugate by washing, the substrate tetramethylbenzidine was added to the wells. RANKL levels were measured by reading the substrate on a standard ELISA plate reader at $450 \mathrm{~nm}$. The detection limit was $1.63 \mathrm{pg} / \mathrm{ml}$. The intra-assay coefficient of variation (CV) was $2-4 \%$ for the serum RANKL measurements, and the inter-assay $\mathrm{CV}$ was $5-8 \%$.

A sandwich ELISA method was also used to assess OPG levels in the serum samples. This assay contained two antibodies against OPG; the biological activity of recombinant human OPG was neutralized by the antibodies. The detection antibody was a goat polyclonal anti-human OPG antibody labeled with biotin (sc-8468; Santa Cruz Biotechnology, Inc.), where during manufacture the goat was immunized with human recombinant OPG (sc-361910Rx; Santa Cruz Biotechnology,
Inc.). The serum levels of OPG were determined on the basis of the protein concentration, according to the manufacturer's instructions (sc-363390; Santa Cruz Biotechnology, Inc.). The detection limit was $2.53 \mathrm{pg} / \mathrm{ml}$. The intra-assay $\mathrm{CV}$ for OPG measurements was $7-9 \%$, and the inter-assay $\mathrm{CV}$ was $<8 \%$.

Statistical analysis. Statistical analyses were performed using SPSS 13.0 (SPSS, Inc., Chicago, IL, USA). Data were expressed as the mean \pm standard deviation (SD) and evaluated using Student's t-test. $\mathrm{P}<0.05$ was considered to indicate a statistically significant difference.

\section{Results}

Serum RANKL and OPG levels. RANKL levels were significantly higher in the intertrochanteric fracture group than in the controls at baseline and 4 weeks following injury $(\mathrm{P}<0.05)$. However, RANKL serum levels were not significantly different between the two groups 8 and 12 weeks following injury ( $P>0.05$ at both time intervals). Serum RANKL levels were higher in the injury group than in the control group at all four time intervals (Fig. 1).

Serum OPG levels were higher in the fracture group than in the control group at all four time points (Fig. 2). Although the OPG levels were significantly higher in the intertrochanteric fracture group than in the controls immediately after and 4 weeks after injury $(\mathrm{P}<0.05$ at both time intervals), there was no significant difference between the two groups 8 and 12 weeks following injury ( $\mathrm{P}>0.05$ at both time intervals; Fig. 2).

RANKL/OPG ratios. The RANKL/OPG ratio was lower in the intertrochanteric fracture group than in the controls at all four time intervals. The ratios were significantly lower immediately after and 4 weeks after injury $(\mathrm{P}<0.05$ at both time intervals), while there was no significant difference between the two groups 8 and 12 weeks after injury (Fig. 3).

\section{Discussion}

In the present study, the serum levels of RANKL and OPG in elderly patients with intertrochanteric fractures and in healthy controls were assessed. To the best of our knowledge, this is the first study to demonstrate a role of the serum levels of RANKL and OPG in elderly patients with intertrochanteric fractures.

The levels of RANKL (Fig. 1) and OPG (Fig. 2) were significantly higher in the fracture group than in the controls immediately after and 4 weeks following injury, while there were no significant differences between the groups 8 and 12 weeks after injury. A previous study has shown that the secretion of RANKL by osteoblasts stimulates osteoclast differentiation (19). Schett et al (20) suggested that serum RANKL levels may be used as a predictor of fracture. Abdallah et al reported an association between high OPG levels and low risk of fracture (21). It has also been suggested that OPG in combination with RANKL may inhibit osteoclast differentiation (22).

In the present study, it was observed that RANKL levels were higher in the intertrochanteric fracture group than in the healthy controls immediately after and 4 weeks after injury (Fig. 1), while the RANKL levels were lower than the OPG levels in the intertrochanteric fracture group at baseline and 


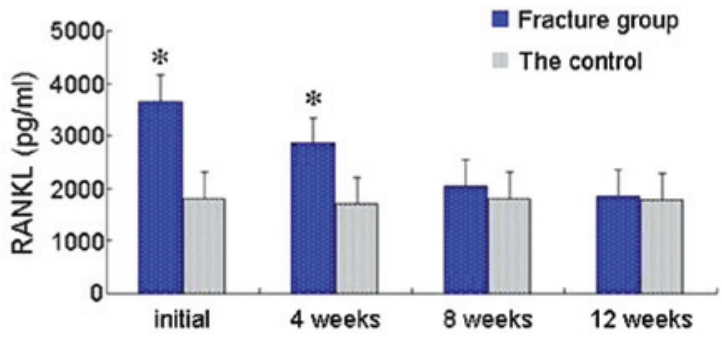

Figure 1. RANKL levels in the intertrochanteric fracture group (blue) were significantly higher $\left({ }^{*} \mathrm{P}<0.05\right)$ than those in the healthy controls (grey) at the time of initial sampling and 4 weeks after injury. Data are presented as the mean \pm SD. RANKL, receptor activator of NF- $\kappa$ B ligand.

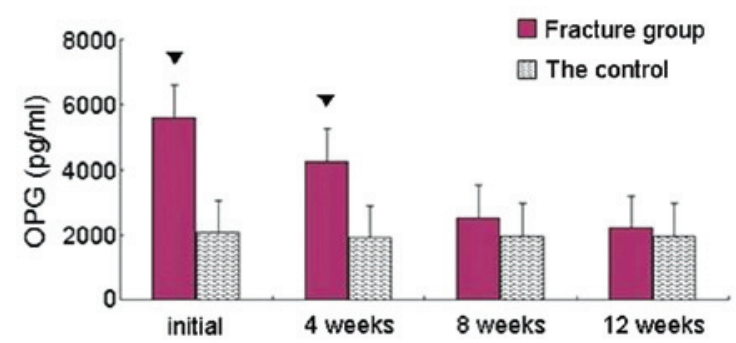

Figure 2. OPG levels in the intertrochanteric fracture group (pink) were significantly higher $\left({ }^{\circ} \mathrm{P}<0.01\right)$ than those in the healthy controls (hashed) immediately after and 4 weeks after injury. Data are presented as the mean $\pm \mathrm{SD}$. OPG, osteoprotegerin .

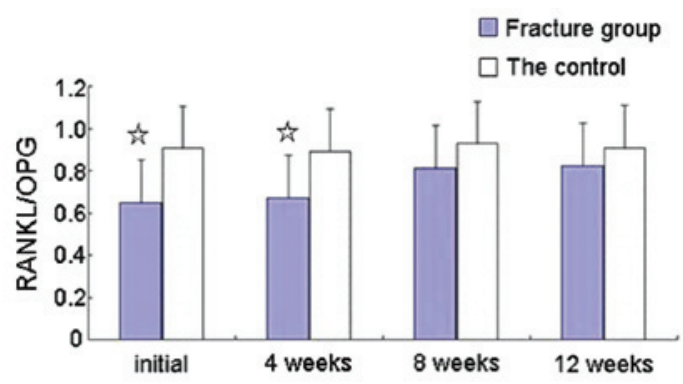

Figure 3. The RANKL/OPG ratio in the intertrochanteric fracture group was significantly lower $\left({ }^{\mathrm{P}}<0.05\right)$ than that in the control group immediately after and 4 weeks after injury. Data are presented as the means \pm SD. RANKL, receptor activator of $\mathrm{NF}-\kappa \mathrm{B}$ ligand; OPG, osteoprotegerin.

4 weeks after injury. These data suggest that the number of osteoblasts increases during healing, particularly immediately after and 4 weeks after injury. As a result, bone formation is likely to dominate the healing process at these two time intervals. Therefore, our results indicate that serum RANKL and OPG may be involved in the healing of intertrochanteric fractures in elderly patients.

Notably, the RANKL/OPG ratios were observed to be significantly lower in the intertrochanteric fracture group than in the normal control subjects immediately after and 4 weeks after injury; and remained lower 8 and 12 weeks following injury (Fig. 3). The RANKL/OPG ratio was lower in the intertrochanteric fracture group than in the normal group, while a slight increase of the ratio with time was noted (Fig. 3). An increasing RANKL/OPG ratio after fracture is considered to tilt the balance of RANKL and OPG toward bone resorptive status (17), since this balance is critically important for bone remodeling and the preservation of bone mass (23). The RANKL-OPG balance may affect osteoclast activity, which is why it has been suggested that the serum RANKL/OPG ratio is a critical factor for determining osteoclast activation (24).

The results of the current study indicate that the RANKL/OPG ratio may be important in the healing process of intertrochanteric fractures in elderly patients. Coetzee and Kruger (25) suggested that this ratio may determine osteoclast activation and affect bone formation, which underlies the potential value of the ratio for predicting fracture healing. Moreover, Tsangari et al (26) demonstrated that the RANKL/OPG ratio predicts fracture healing (26). The present study indicates that bone formation mainly occurs during the first 8 weeks, and particularly during the first 4 weeks following injury; bone remodeling mainly occurs 8 and 12 weeks following injury. Future studies are required in order to determine why the RANKL and OPG levels are higher at the initial time intervals after intertrochanteric fracture than their levels in healthy subjects. Since fracture healing is a complex process, the changes in RANKL and OPG levels may also be influenced by other factors, which will be investigated in future studies.

In conclusion, high serum levels of RANKL and OPG were detected in patients with intertrochanteric fractures compared with healthy controls. The present study demonstrated that the RANKL/OPG ratio was lower in the intertrochanteric fracture group than in the healthy controls. These observations provide important information regarding the healing process of intertrochanteric fractures in the elderly at a molecular level. In the present study, the serum RANKL/OPG ratio has been suggested to contribute to the modulation of fracture repair. Consequently, these findings may lead to novel therapeutic interventions for nonunion of intertrochanteric fractures.

\section{Acknowledgements}

The authors would like to thank Dr Li-Juan Guo from the Molecular Laboratory of Peking University (Beijing, China) for advice regarding the setup of the ELISA experiment, and Dr Guo-Xing Li from the Department of Public Health (Peking University) for assistance in statistical analysis.

\section{References}

1. Kesmezacar H, Oğüt T, Bilgili MG, Gökay S and Tenekecioğlu Y: Treatment of intertrochanteric femur fractures in elderly patients: internal fixation or hemiarthroplasty. Acta Orthop Traumatol Turc 39: 287-294, 2005 (In Turkish).

2. Angelini M, McKee MD, Waddell JP, Haidukewych G and Schemitsch EH: Salvage of failed hip fracture fixation. J Orthop Trauma 23: 471-478, 2009.

3. Talmo CT and Bono JV: Treatment of intertrochanteric nonunion of the proximal femur using the S-ROM prosthesis. Orthopedics 31: 125, 2008.

4. Dhammi I, Jain A, Singh A, Rehan-Ul-Haq, Mishra P and Jain S: Primary nonunion of intertrochanteric fractures of femur: an analysis of results of valgization and bone grafting. Indian J Orthop 45: 514-519, 2011.

5. Looker AC, Bauer DC, Chesnut CH 3rd, Gundberg CM, Hochberg MC, Klee G, Kleerekoper M, Watts NB and Bell NH: Clinical use of biochemical markers of bone remodeling: current status and future directions. Osteoporos Int 11: 467-480, 2000. 
6. Kurban S and Mehmetoglu I: Osteoprotegerin, RANK and RANK ligand. Turk J Biochem 34: 178-184, 2007.

7. Stejskal D, Bartek J, Pastorková R, Růzicka V, Oral I, Horalík D: Osteoprotegerin, RANK, RANKL. Biomed Pap Med Fac Univ Palacky Olomouc Czech Repub 145: 61-64, 2001.

8. Khosla S: Minireview: the OPG/RANKL/RANK system. Endocrinology 142: 5050-5055, 2001.

9. Kostenuik PJ and Shalhoub V: Osteoprotegerin: a physiological and pharmacological inhibitor of bone resorption. Curr Pharm Des 7: 613-635, 2001.

10. Bucay N, Sarosi I, Dunstan CR, Morony S, Tarpley J, Capparelli C, Scully S, Tan HL, Xu W, Lacey DL, Boyle WJ and Simonet WS Osteoprotegerin-deficient mice develop early onset osteporosis and arterial calcification. Genes Dev 12: 1260-1268, 1998.

11. Gravallese EM, Manning C, Tsay A, Naito A, Pan C, Amento E and Goldring SR: Synovial tissue in rheumatoid arthritis is a source of osteoclast differentiation factor. Arthritis Rheum 43 250-258, 2004

12. Hafbauer LC and Schoppet M: Clinical implications of the osteoprotegerin/RANKL/RANK system for bone and vascular diseases. JAMA 292: 490-495, 2004.

13. Masi L, Simonini G, Giani T, Del Monte F, Giani T, Cimaz R, Vierucci S, Brandi ML and Falcini F: Osteoprotegerin (OPG)/ RANKL system in juvenile idiopathic arthitis: is there a potential modulating role for OPG/RANKL in bone injury? J Rheumatol 31: 986-991, 2004.

14. Blair JM, Zheng Y and Dunstan CR: RANK ligand. Int J Biochem Cell Biol 39: 1077-1081, 2007.

15. Chamoux E, Houde N, L'Eriger K and Roux S: Osteoprotegerin decreases human osteoclast apoptosis by inhibiting the TRAIL pathway. J Cell Physiol 216: 536-542, 2008.

16. Boyce BF and Xing L: Function of RANKL/RANK/OPG in bone modeling end remodeling. Arch Biochem Biophys 473: $139-146,2008$

17. Lacey DL, Timms E, Tan HL, et al: Osteoprotegerin ligand is a cytokine that regulates osteoclast differentiation and activation. Cell 93: 165-176, 1998.
18. Simonet WS, Lacey DL, Dunstan CR, et al: Osteoprotegerin: a novel secreted protein involved in the regulation of bone density. Cell 89: 309-319, 1997.

19. Son A, Kim MS, Jo H, Byun HM and Shin DM: Effects of inositol 1,4,5-triphosphate on osteoclast differentiation in RANKL-induced osteoclastogenesis. Korean J Physiol Pharmacol 16: 31-36, 2012.

20. Schett G, Kiechl S, Redlich K, Oberhollenzer F, Weger S, Egger G, Mayr A, Jocher J, Xu Q, Pietschmann P, Teitelbaum S, Smolen J and Willeit J: Soluble RANKL and risk of non-traumatic fracture. JAMA 291: 1108-1113, 2004.

21. Abdallah BM, Stilgren LS, Nissen N, Kassem M, Jørgensen HR and Abrahamsen B: Increased RANKL/OPG mRNA ratio in iliac bone biopsies from women with hip fractures. Calcif Tissue Int 76: 90-97, 2005.

22. Yasuda H, Shima N, Nakagawa N, Yamaguchi K, Kinosaki M, Goto M, Mochizuki SI, Tsuda E, Morinaga T, Udagawa N, Takahashi N, Suda T and Higashio K: A novel molecular mechanism modulating osteoclast differentiation and function. Bone 25: 109-113, 1999.

23. Wasilewska A, Rybi-Szuminska A and Zoch-Zwierz W: Serum RANKL, osteoprotegerin (OPG), and RANKL/OPG ratio in nephrotic children. Pediatr Nephrol 25: 2067-2075, 2010.

24. Hofbauer LC, Khosla S, Dunstan CR, Lacey DL, Boyle WJ and Riggs BL: The roles of osteoprotegerin and osteoprotegerin ligand in the paracrine regulation of bone resorption. $\mathrm{J}$ Bone Miner Res 15: 2-12, 2000.

25. Coetzee $\mathrm{M}$ and Kruger MC: Osteoprotegerin-receptor activator of nuclear factor-kappaB ligand ratio: a new approach to osteoporosis treatment? South Med J 97: 506-511, 2004

26. Tsangari H, Findlay DM, Kuliwaba JS, Atkins GJ and Fazzalari NL: Increased expression of IL-6 and RANK mRNA in human trabecular bone from fragility fracture of the femoral neck. Bone 35: 334-342, 2004. 